Fent osecond- I aser - encoded di st $r$ i but ed- $f$ eedback col or center I aser in I ithi umfluoride single cryst al $\mathrm{s}$

\begin{tabular}{|l|l|}
\hline 著者 & $\begin{array}{l}\text { Kawamur a Ken- i chi, H r ano Masahi ro, Kur obor i } \\
\text { Toshi o, Takami zu Dai zyu, Kam ya Toshi o, Hosono } \\
\text { H deo }\end{array}$ \\
\hline $\begin{array}{l}\text { j our nal or } \\
\text { publ i cat i on t i t l e }\end{array}$ & Appl i ed Physi cs Let ter s \\
\hline vol une & 84 \\
\hline number & 3 \\
\hline page r ange & $311-313$ \\
\hline year & 2004 01- 19 \\
\hline URL & ht t p: //hdl . handl e. net /2297/3778 \\
\hline
\end{tabular}




\title{
Femtosecond-laser-encoded distributed-feedback color center laser in lithium fluoride single crystals
}

\author{
Ken-ichi Kawamura ${ }^{a)}$ and Masahiro Hirano \\ Hosono Transparent Electro-Active Materials (TEAM) Project, Exploratory Research for Advanced \\ Technology (ERATO), Japan Science and Technology, KSP C-1232, Sakato 3-2-1, Takatsu-ku, \\ Kawasaki 213-0012, Japan \\ Toshio Kurobori \\ Department of Materials Science and Engineering, Kanazawa University, Kakuma, \\ Kanazawa 920-1192, Japan \\ Daizyu Takamizu, Toshio Kamiya, and Hideo Hosono \\ Materials and Structures Laboratory, Tokyo Institute of Technology, Nagatsuta, Midori-ku, \\ Yokohama 226-8503, Japan
}

(Received 25 September 2003; accepted 19 November 2003)

\begin{abstract}
Laser-active $\mathrm{F}_{2}$ centers were produced in lithium fluoride $(\mathrm{LiF})$ at a concentration of 2 $\times 10^{18} \mathrm{~cm}^{-3}$ by irradiating focused femtosecond (fs) laser pulses from a mode-locked titanium sapphire laser (wavelength $\sim 800 \mathrm{~nm}$, emission pulse duration $\sim 100 \mathrm{fs}$ ). This technique was used to write waveguides embedded in LiF crystals. A refractive index change estimated from a guide propagation method was approximately $+1 \%$ at a wavelength of $633 \mathrm{~nm}$. Refractive index-modulated volume-type gratings were also encoded inside LiF crystals by a single interfered fs laser pulse. The distributed feedback laser structure was fabricated using the gratings thus encoded, which exhibited a room-temperature $F_{2}$-color center laser oscillation at $707 \mathrm{~nm}$. This demonstrates a DFB color center laser operating at room temperature utilizing photon written, permanent Bragg gratings. (C) 2004 American Institute of Physics. [DOI: 10.1063/1.1640784]
\end{abstract}

Typically, materials used for optical applications have wide optical band gaps such as $\mathrm{SiO}_{2}$ glass and sapphire. Due to the wide band gaps, it is difficult to process these materials with conventional light. A femtosecond (fs) laser defies this limitation since the fs pulse can generate light pulses with extremely high peak power densities, e.g., up to about several hundreds $\mathrm{TW} / \mathrm{cm}^{2}$, from the regenerative amplifier system of a mode-locked titanium sapphire. This high power fs-laser pulse may cause strong optical interaction with materials through nonlinear effects. Therefore, it is expected to process any materials including optically transparent dielectrics.

It has been reported that almost any kind of material including photoinsensitive transparent dielectrics and metals can be processed. ${ }^{1,2}$ The pulse generated from a mode-locked pulsed laser has nearly perfect temporal coherency, which creates the possibility of developing more advanced optical processes. We have developed a hologram technique to encode nonerasable structures by a single interfered infrared fs laser pulse, ${ }^{3,4}$ in which holographic gratings are encoded on the surface of various materials such as pure $\mathrm{SiO}_{2}$ glass, sapphire, and diamond. The technique was also used to fabricate more advanced structures at the nanometer scale such as cross gratings and two-dimensional nanohole structures using a double exposure technique. ${ }^{5}$ Furthermore, we reported that chirped fs laser pulses can machine deep inside transparent materials several millimeters from the surface. ${ }^{6}$ Three-dimensional volume-type grating arrays were successfully encoded inside pure silica glass with a chirped fs laser

${ }^{a)}$ Electronic mail: kawamura@lucid.rlem.titech.ac.jp pulse. These results suggest that this technique has the potential to integrate optical device components inside transparent materials, which will provide the possibility for active optical devices.

In this letter, we report that an active optical device, a distributed-feedback (DFB) color-center laser, is fabricated by the holographic encoding method using an infrared fs laser. Laser active color centers, permanent gratings, and waveguide structures are written deep in lithium fluoride (LiF) crystals. The fabricated DFB laser structure oscillates at room temperature.

$\mathrm{LiF}$ has been examined for room-temperature color center laser since it has good physical and chemical properties and the formed laser-active color centers have high photothermal stability. ${ }^{7-10}$ The color centers, such as $\mathrm{F}_{2}$, and $\mathrm{F}_{3}^{+}$ centers in $\mathrm{LiF}$ are promising for visible laser action. Recently, Montecchi ${ }^{11}$ reported that the formation of color centers caused an increase in the refractive index. They also successfully fabricated active channel waveguides by electron-beam (e-beam) lithography.

High-energy lithographic techniques such as e-beam, ion implantation, $\mathrm{x}$ - and $\gamma$-ray irradiation are applicable to materials with large optical band gaps including $\mathrm{LiF}$, but it is difficult to define fine patterns such as grating structures and three-dimensional optical circuits. Moreover, vacuum chambers and long exposure times are generally required for these purposes. High-power density fs pulses would overcome these drawbacks, i.e., it can simultaneously fabricate laser active color center and holographic grating structures on a tabletop in an ambient atmosphere.

A regenerative amplified fs pulse from a mode-locked 
(a)

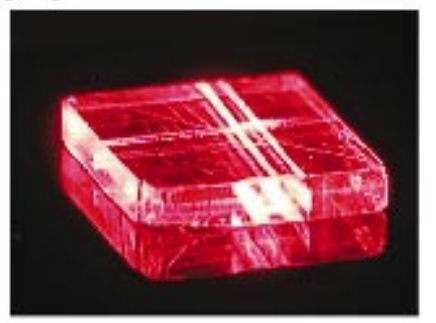

(b)
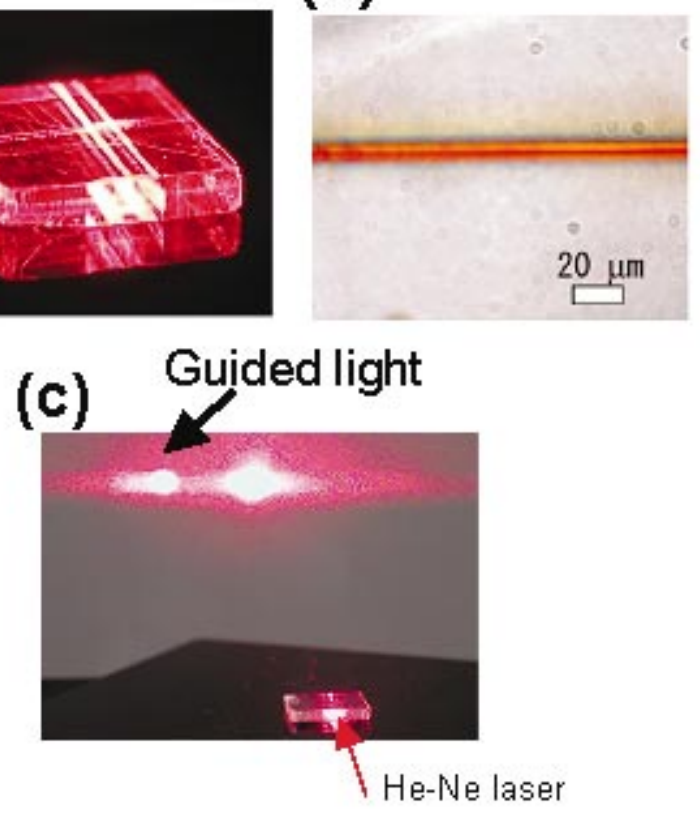

FIG. 1. (Color) (a) Fluorescence image of embedded waveguides under 450 nm light excitation; (b) an optical microscope image of the waveguide encoded with fs pulses; (c) image showing coupling of $\mathrm{He}-\mathrm{Ne}$ laser beam $(\lambda$ : $633 \mathrm{~nm}$ ) with the waveguide. Left spot on the screen is a beam emerging from the waveguide.

titanium sapphire laser (wavelength: $800 \mathrm{~nm}$, pulse duration: $100 \mathrm{fs}$, repetition: $10 \mathrm{~Hz}$ ) was employed in this study. Undoped LiF single crystals (impurities; calcium 7, iron 1, and magnesium 1 wt ppm) were used. To examine the formation of color centers, fs pulses (70 $\mu \mathrm{J} /$ pulse, $100 \mathrm{fs}$ ) that were focused through a convex lens $(f=60 \mathrm{~mm})$ irradiated a $\mathrm{LiF}$ crystal. The sample was moved using a stepping-motorcontrolled stage synchronized with the laser pulse repetition rate. Each point was irradiated with 50 pulses. The induced optical absorption spectrum was obtained by subtracting the spectrum before irradiating from that after irradiating. Two absorption bands peaking at 4.9 and $2.8 \mathrm{eV}$ were clearly seen in the difference absorption spectrum. The former originates from $\mathrm{F}$ centers and the latter from the $\mathrm{F}_{2}$ and $\mathrm{F}_{3}^{+}$-centers. The estimated concentration of the $\mathrm{F}_{2}$ color center was 2 $\times 10^{18} \mathrm{~cm}^{-3}$ using Smakula's expression ${ }^{12}$ and a reported value of the oscillator strength. ${ }^{13}$ Although the optical band
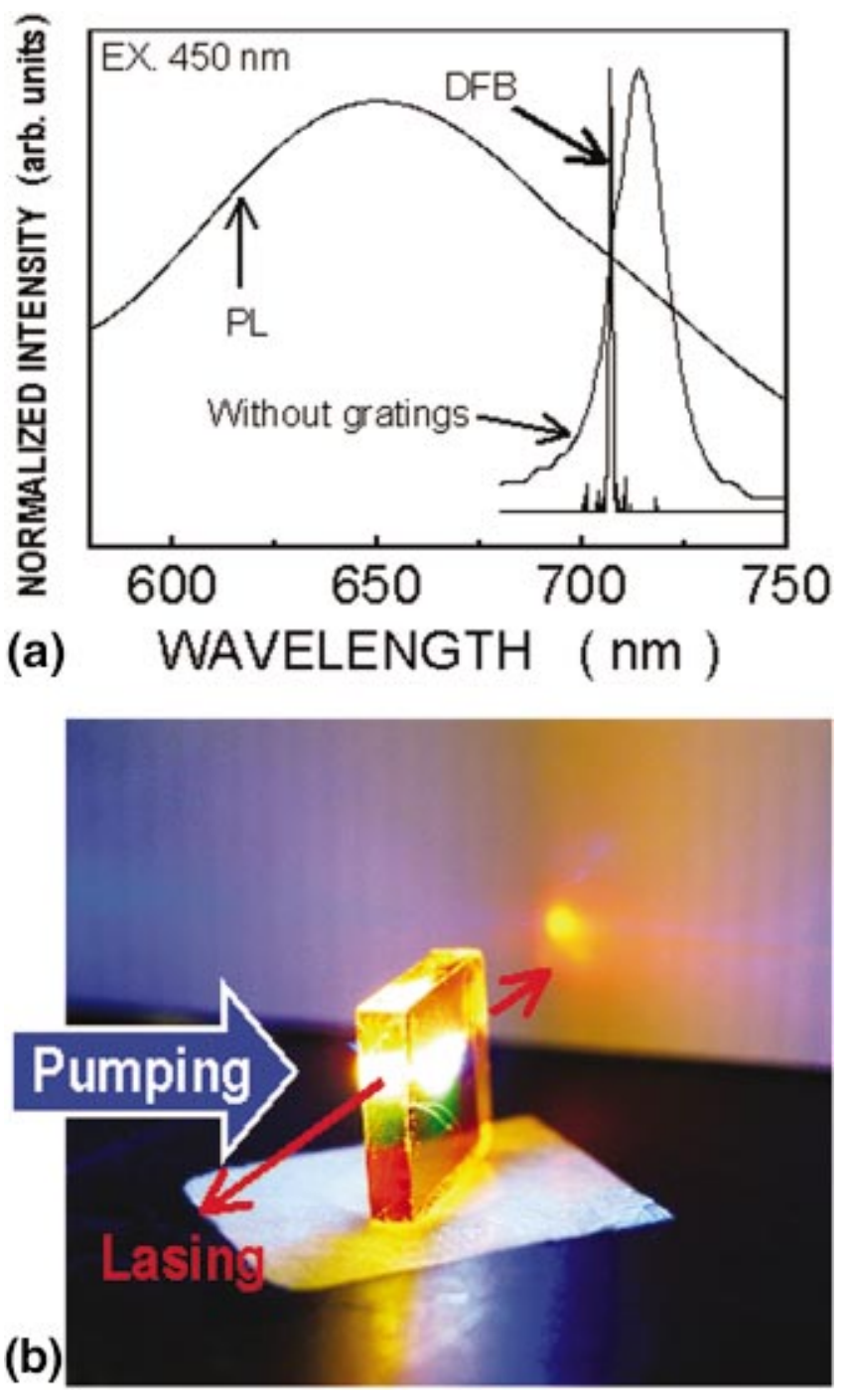

FIG. 3. (Color) (Top) emission spectra from the DFB laser structure and a sample without gratings. Conventional PL is also shown for comparison. Emission spectra are obtained by pumping the $\mathrm{F}_{2}$ center with an OPO laser system at $450 \mathrm{~nm}$ (repetition frequency: $10 \mathrm{~Hz}$, pulse duration: $\sim 10 \mathrm{~ns}$ ). The line spacing of the grating is $\sim 510 \mathrm{~nm}$. Theoretically expected DFB oscillation wavelength from the line spacing employing second-order diffraction is $710 \mathrm{~nm}$. After encoding the gratings, additional x-ray irradiation was conducted. (Bottom) image showing the DFB laser oscillation. A beam spot emerging from the crystal is seen on the screen. (a)

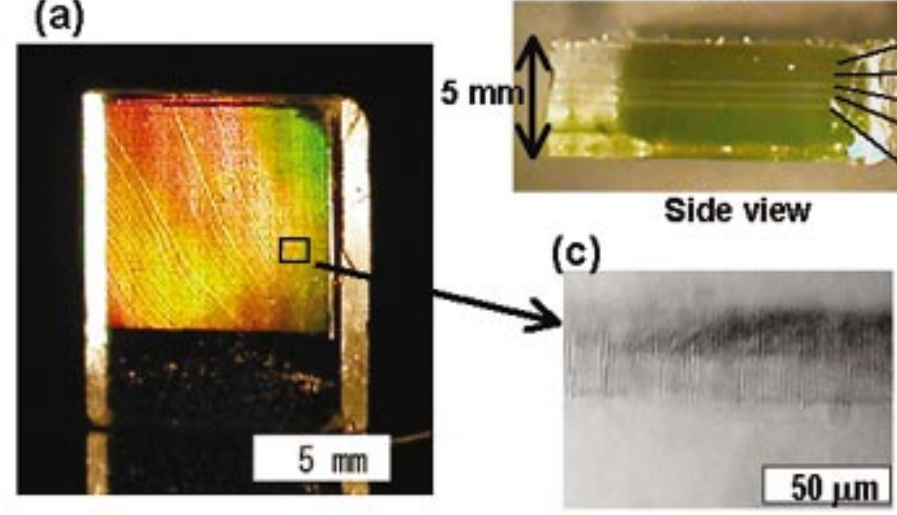

FIG. 2. (Color) Images showing five layers of twodimensional micrograting arrays encoded inside a $\mathrm{LiF}$ crystal. Grating structure was encoded inside LiF crystal using a single interference laser pulse with $\sim 100 \mu \mathrm{J} /$ pulse energy and $\sim 500 \mathrm{fs}$ duration. 
gap of $\mathrm{LiF}(14 \mathrm{eV})$ is much larger than the photon energy $(1.5 \mathrm{eV})$, high-density color centers are produced in LiF by the infrared fs laser pulses. These results demonstrate that the fs laser pulse can be used to ionize and process wide bandgap materials such as LiF instead of other conventional ionizing beams.

Figure 1(a) shows an image of waveguides written in a $\mathrm{LiF}$ crystal, (b) its magnified image, and (c) an image exhibiting coupling of the waveguide with a helium neon $(\mathrm{He}-\mathrm{Ne})$ laser beam $(\lambda: 633 \mathrm{~nm})$. Irradiating line-shape laser pulses focused through a cylindrical lens $(f=50 \mathrm{~mm})$ in the sample that was moved at a rate of $50 \mu \mathrm{m} / \mathrm{s}$ fabricated the waveguides. The beam size was $\sim 10 \times 3000 \mu \mathrm{m}^{2}$ and the beam power at the sample was $\sim 800 \mu \mathrm{J} /$ pulse. Figure 1(a) shows the sample excited by $532 \mathrm{~nm}$ illumination. Strong red luminescence (arising from the $\mathrm{F}_{2}$ centers) from the embedded waveguides is clearly observed. Green luminescence of the $\mathrm{F}_{3}^{+}$centers was also observed when excited by $450 \mathrm{~nm}$ light. The refractive index change induced by the color centers was estimated by detecting a critical coupling angle with $\mathrm{He}-\mathrm{Ne}$ laser light. In Fig. 1(c), $\mathrm{He}-\mathrm{Ne}$ laser light was coupled from the end of the waveguide at various incident angles. A light spot emitted from the waveguide was projected on the left-hand side of the screen and the estimated refractive index change was about $+1 \%$ at $633 \mathrm{~nm}$. It is noteworthy that the waveguide structure was stable even at room temperature and remarkably was not erased even when irradiating with intense optical light.

It is also possible to three-dimensionally align the nonerasable gratings in LiF. Figure 2 shows images of a $\mathrm{LiF}$ crystal in which five layers of two-dimensional micrograting arrays were encoded by the interference of two fs pulses split from a single pulse. Details of the experimental setup are reported in Ref. 14. The pulse energy and duration were $\sim 100 \mu \mathrm{J} /$ pulse and $\sim 500 \mathrm{fs}$, respectively. An image of a side view (b) indicates that gratings are encoded inside the sample at depths of $40-540 \mu \mathrm{m}$ from the incident surface. The diameter of the grating spot was $\sim 40 \mu \mathrm{m}$ and the spacing between adjacent gratings was $1 \mu \mathrm{m}$. Irradiating with intense optical light did not erase the encoded grating structure.

We then fabricated a DFB color center laser structure using nonerasable gratings. DFB laser action for $\mathrm{F}_{2}$ center in $\mathrm{LiF}$ has already been reported, ${ }^{10}$ but the study used transient grating structures since there was not a method that embedded permanent gratings in LiF. Here, a one-dimensional array of microgratings, $40 \mu \mathrm{m}$ in diameter, were written in a 10 $\mathrm{mm}$ line at a depth of $\sim 100 \mu \mathrm{m}$ (grating strings). Since we aimed at lasing from the $\mathrm{F}_{2}$ center, the designed line spacing of the gratings was $510 \mathrm{~nm}$ and was achieved by adjusting the laser pulse crossing angle to $102^{\circ}$, which corresponds to the theoretically expected DFB oscillation wavelength of 710 $\mathrm{nm}$ using second order diffraction. After encoding the gratings, x-rays $(\mathrm{Cu} \mathrm{K} \alpha, 35 \mathrm{keV}-25 \mathrm{~mA}, 3 \mathrm{~h})$ further irradiated the sample to earn enough gain for DFB laser action through an increase in the uniform concentration of $F_{2}$ center.
A side-pumping configuration was employed using an intense 450-nm laser pulse from an optical parametric oscillator (OPO) (repetition frequency: $10 \mathrm{~Hz}$, pulse duration: 10 ns). A cylindrical lens focused the laser pulse at the grating line with an intensity of $0.8 \mathrm{~mJ} / \mathrm{pulse}$. A charge coupled device (CCD) spectrometer measured the outputs from the DFB laser. Figure 3 (top) shows an emission spectrum from the grating strings in the LiF crystal. For comparison, the spectra from an $\mathrm{x}$-ray irradiated $\mathrm{LiF}$ without a grating structure excited by the intense OPO laser pulse and by a xenon lamp (conventional photoluminescence) are shown.

When the intense laser pulse excites the sample without the gratings, the emission band becomes narrower than that of the conventional PL, indicating the occurrence of amplified spontaneous emission. A much narrower, intense emission is clearly observed at $707 \mathrm{~nm}$ in the x-ray irradiated LiF that has the grating strings. The observed oscillation wavelength is consistent with the theoretically expected value 710 $\mathrm{nm}$ and the linewidth is less than the resolution of our measurement system $(1 \mathrm{~nm})$. This narrow emission spectrum has not been observed without gratings.

Figure 3 (bottom) shows an image of the DFB laser action. An intense beam spot emerging from the crystal is distinctly observed on the screen, which demonstrates that the color center laser oscillates and that the nonerasable gratings encoded form a DFB laser structure, which operates at room temperature.

In summary, we successfully produced laser-active color centers and fabricated optical waveguides and microgratings deep inside LiF crystals by femtosecond laser pulses. We also successfully demonstrated that simultaneous formation of a color center and waveguide with interfered-fs-laserinduced microgratings produced an oscillating DFB color laser. This work demonstrates the applicability of our fs laser pulse interference technique for practical integrated optical devices.

${ }^{1}$ E. N. Glezer, M. Milosavljevic, L. Huang, R. J. Finlay, T.-H. Her, J. P. Callan, and E. Mazur, Opt. Lett. 21, 2023 (1996).

${ }^{2}$ E. N. Glezer and E. Mazur, Appl. Phys. Lett. 71, 882 (1997).

${ }^{3}$ K. Kawamura, N. Sarukura, M. Hirano, and H. Hosono, Appl. Phys. B: Lasers Opt. 71, 119 (2000); Jpn. J. Appl. Phys., Part 2 39, L767 (2000).

${ }^{4}$ K. Kawamura, N. Sarukura, M. Hirano, and H. Hosono, Appl. Phys. Lett. 78, 1038 (2001).

${ }^{5}$ K. Kawamura, N. Sarukura, M. Hirano, N. Ito, and H. Hosono, Appl. Phys. Lett. 79, 1228 (2001).

${ }^{6}$ K. Kawamura, M. Hirano, T. Kamiya, and H. Hosono, Appl. Phys. Lett. 81, 1137 (2001).

${ }^{7}$ H. E. Gu, L. F. Wan, and H. S. Guo, Opt. Commun. 70, 141 (1989).

${ }^{8}$ T. T. Basiev, S. B. Mirov, and V. V. Osiko, IEEE J. Quantum Electron. 24, 1052 (1988).

${ }^{9}$ T. Tsuboi and H. E. Gu, Appl. Opt. Commun. 55, 277 (1985).

${ }^{10}$ T. Kurobori, K. Inabe, and N. Takeuchi, J. Phys. D 16, L121 (1983).

${ }^{11}$ M. Montecchi, E. Nichelatti, A. M. Mancini, and R. M. Montereali, J. Appl. Phys. 86, 1 (1999).

${ }^{12}$ W. B. Fowler, in Physics of Color Centers, edited by W. B. Fowler (Academic, New York, 1968), Chap. 2.

${ }^{13}$ T. I. Garbovskaja, L. K. Kants, and G. V. Leja, Bull. Latv. Acad. Sci. Ser. Phys. Tech. Sci. 6, 44 (1980).

${ }^{14}$ K. Kawamura, N. Ito, N. Sarukura, M. Hirano, and H. Hosono, Rev. Sci. Instrum. 73, 1711 (2002). 